Urologe 2018·57:1431-1432

https://doi.org/10.1007/s00120-018-0811-3

(c) Springer Medizin Verlag GmbH, ein Teil von Springer Nature 2018

CrossMark

\author{
W. Bühmann ${ }^{1,2}$ \\ 'Berufsverband der Deutschen Urologen e. V., Sylt OT Keitum, Deutschland \\ ${ }^{2}$ Sylt OT Morsum, Deutschland
}

\title{
Sexuell übertragbare Infektionen - seit der Antike aktuell
}

Liebe Kolleginnen und Kollegen,

seit Entdeckung genitaler Warzen in der römischen, griechischen und ägyptischen Antike begleiten sexuell übertragbare Infektionen (STI) - zunächst mehr gesellschaftlich, erst in der jüngeren Geschichte rückten sie mit detaillierten Analysen zur Ätiologie, Diagnostik und Therapieoptionen auch in den ärztlichen Fokus - die Menschen unterschiedlich intensiv in verschiedenen Kulturkreisen. Erster Höhepunkt war die Entdeckung der Syphilis als STI um 1500 und die damit assoziierten sexuellen Gewohnheiten bis heute.

Zwei Beispiele abseits der Urologie mögen uns die aktuelle Bedeutung der STI belegen: Erst Ende der 1930er-Jahre konnte die Ätiologie der seit Jahrtausenden bekannten Hepatitiden als viral ausgelöst und sexuell übertragbar geklärt werden, trotz der Impfung fordert allen voran die Hepatitis B unter den weltweit 390 Mio. Virusträgern jährlich 1,5 Mio. Todesopfer durch die Endstadien Leberzirrhose und Leberzellkarzinom.

Seit 1959 bekannt, entwickelte sich das „human immundeficiency virus“ (HIV) sich zur weltweit bedeutendsten STI, die Erstübertragung wird einem sexuellen Kontakt zwischen einem Menschenaffen und einem Mann zugeschrieben. Weltweit jährlich 1,2 Mio. (insgesamt bisher39 Mio.) Tote und jährlich 2 Mio. Neuinfizierte belegen die Dimension dieser STI. In Südafrika starb eine ganze Generation junger Männer und Frauen an AIDS, sodass deren Kinder von Großeltern erzogen und sozialisiert wurden gesellschaftlich und volkswirtschaftlich wohl der traurige Höhepunkt aller STI.
Erste Beobachtungen um 1800 machten deutlich, dass verheiratete Frauen und Prostituierte vermehrt am Zervixkarzinom starben, Nonnen jedoch nicht Beginn einer systematischen Forschung zum humanen Papillomvirus (HPV), die über die Erkenntnis, dass praktisch alle Zervixkarzinome durch HPV erzeugt werden, zur Entwicklung einer wirksamen Impfung durch den deutschen Arzt und Forscher Harald zur Hausen führte, die 2008 mit dem Nobelpreis für Medizin gewürdigt wurde. Im weiteren Verlauf konnte neben der häufigen Infektion mit Condylomata acuminata auch das onkogene HPV-Risikopotential von Anal-, Vulva-, Vaginal-, Penis- und Oropharynxkarzinomen bewiesen werden.

Nach der initial 2007 empfohlenen Impfung für Mädchen hat sich die Ständige Impfkommission (STIKO) erst 2018 zur Impfempfehlung auch für Jungs entschlossen, also mit 10 Jahren Versäumnis. Allein die Logik gebietet, eine Impfung gegen STI selbstverständlich beiden Geschlechtern zu empfehlen, wie es in Australien, Kanada, USA, Österreich und England bereits vor Jahren propagiert wurde. Die bisherige, traurig niedrige Impfquote von etwa $40 \%$ der deutschen Mädchen unter Aussparung homosexueller Männer hat dazu geführt, dass die zur Herdenimmunität und damit möglichen Elimination HPV-assoziierter Erkrankungen notwendige Impfquote von $80 \%$ nicht erreicht wurde. Somit sollte prioritäres Ziel aller Ärzte sein, alle Eltern und Kinder über die Notwendig-

Aus Gründen der besseren Lesbarkeit wird in diesem Beitrag überwiegend das generische Maskulinum verwendet. Dies impliziert immer beide Formen, schließt also die weibliche Form mit ein. 
keit der HPV-Impfung aufzuklären und sie zur Impfung zu bewegen.

Unser vorliegendes Heft spannt im Leitthema "STI“ den Bogen von einer medizinhistorischen Betrachtung der Syphilis von Gernot Bonkat aus Basel: „Syphilis und Heinrich VIII. - Eine Erkrankung schreibt Weltgeschichte" über eine Darstellung aktuell relevanter STI aus der berufenen Feder von Celina Hein und Florian Wagenlehner von der Gießener Universitätsklinik: „Übersicht zur aktuellen Diagnostik und Therapie der sexuell übertragbaren Infektionen“, der frauenärztlichen Perspektive auf HPV von Monika Schmidmayr und Frauke Kleinsorge aus der Gynäkologischen Universitätsklink der Technischen Universität München: „HPV-Infektion bei Frauen - Diagnostik, Therapie und Bedeutung der Impfung “, die urologische Bedeutung von HPV von Peter Schneede aus der Urologischen Klinik Memmingen: „HPV-Krankheitslast der Männer HPV-Vorsorge wird Männersache "zu einem für Urologen eher neuen Aspekt der HPV-assoziierten HNO-Tumoren von Steffen Wagner, HNO-Uniklinik Gießen, Jens Peter Klußmann, HNO-Uniklinik Köln et al. „Das HPV-getriebene Oropharynxkarzinom - Inzidenz-Trends, Diagnose und Therapie“.

Mit diesem für Sie, liebe Kolleginnen und Kollegen, hoffentlich interessanten Potpourri möchte ich einen Appell verbinden: Entdecken Sie das Potential, Ihre urologische Kompetenz einzubringen, neben den eindrucksvollen uroonkologischen Erfolgen Ihr Leistungsportfolio in der Prävention durch Jungensprechstunde und HPV-Jungsimpfung, Diagnostik und Therapie der in deutlich höherer Inzidenz als bei allen onkologischen Entitäten auftretenden STI zu intensivieren.
Mit herzlichen Grüßen wünsche ich Ihnen friedvolle Weihnachtstage

Ihr

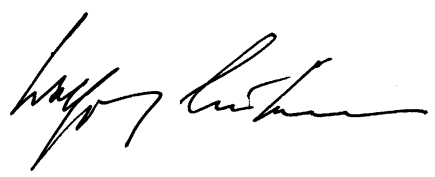

Wolfgang Bühmann

\section{Korrespondenzadresse}

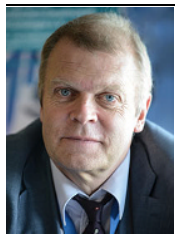

Dr. med. W. Bühmann Terpstig 55, 25980 Sylt OT Morsum, Deutschland post@urologie-sylt.de

Interessenkonflikt. W. Bühmann gibt an, dass kein Interessenkonflikt besteht.

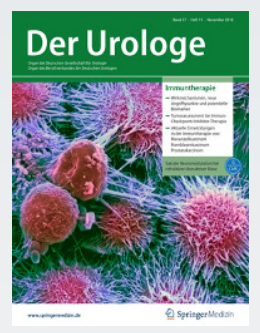

Auf SpringerMedizin.de erhalten Sie Zugang zu allen elektronisch verfügbaren Ausgaben und dem CME-Angebot Ihrer Zeitschrift - unabhängig davon, seit wann Sie Der Urologe abonniert haben. Außerdem können Sie die Zeitschrift mit dem E-Paper auch bequem auf Ihrem Tablet lesen.

So einfach erhalten Sie Zugang zum Online Archiv Registrieren Sie sich einmalig auf www.springermedizin.de/register

Geben Sie dabei Ihre Einheitliche Fortbildungsnummer (EFN) an.

- Ihr Benutzername entspricht Ihrer E-Mail-Adresse, Ihr Passwort können Sie frei wählen und später jederzeit unter „Mein Profil“ ändern.

- Falls Sie bereits ein (Print-) Abonnement bei uns haben, geben Sie bei der Registrierung die Lieferadresse Ihrer Zeitschrift an. Damit wird Ihr Abo-Zugang auf springermedizin.de freigeschaltet.
Sind Sie bereits bei SpringerMedizin.de registriert? Dann wird Ihr Zeitschriftenabonnement automatisch Ihrem Online-Nutzerkonto hinzugefügt. Sollten die Angaben Ihres Online-Accounts nicht eindeutig mit den Angaben Ihres Zeitschriften-Abonnements übereinstimmen, kann die Zuordnung nicht sicher erfolgen. In diesem Fall und bei allen anderen Fragen zum Online-Zugang kontaktieren Sie bitte unseren Kundenservice unter: Kundenservice@springermedizin.de

Telefonisch erreichen Sie die Hotline montags bis freitags von 9.00 bis 17.00 Uhr kostenfrei unter 0800-77 80777 sowie gebührenpflichtig aus dem Ausland unter $\hookleftarrow+4930884293600$ 